\title{
Correlación entre los niveles de glutatión peroxidasa, un marcador de estrés oxidativo, y la presentación clínica del dengue
}

\author{
Elsa Marina Rojas ${ }^{1}$, Fredi Alexander Díaz-Q uijano ${ }^{1}$, \\ Carolina Coronel-Ruiz ${ }^{2}$, Ruth Aralí Martínez-Vega ${ }^{1}$, \\ Ernesto Rueda ${ }^{3}$, Luis Angel Villar-Centeno ${ }^{1}$. \\ Association between glutathione \\ peroxidase levels and clinical \\ manifestations of dengue
}

Background: Glutathione peroxidase (GP) can be used as a marker of oxidative stress in infectious diseases. Aim: To evaluate the association between the levels of glutathione peroxidase (GP) and the manifestations and complications of dengue. Patients and Methods: Between April 2003 and December 2004, 161 patients with dengue were prospectively evaluated. In the first evaluation, within 48 and 96 hours of disease onset, a plasma sample was obtained to measure the GP levels. The association between GP levels, clinical manifestations and complications was evaluated during the follow up. Results: Mean GP values were $1198 \mathrm{U} / \mathrm{L}$ (95\% confidence interval 1089-1306). Values greater than $1200 \mathrm{U} / \mathrm{L}$ were associated with headache, arthralgias and increased heart rate. There was a negative association between GP levels and serum triglycerides. During follow up, patients with GP $>1200$ U/L had a higher frequency of spontaneous hemorrhages. In a logistic regression analysis arthralgias, fever and increased heart rate, were independently associated with levels > 1200 U/L Conclusions: GP levels was associated to some of the manifestations of dengue. This finding suggests that the intensity of oxidative stress can influence the clinical presentation of dengue (Rev Méd Chile 2007; 135: 743-50).

(Key w ords: Arthralgia; Dengue; Fever; Glutathione peroxidase)

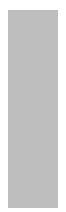

Recibido el 26 de julio, 2006. Aceptado el 30 de noviembre, 2006.

Financiación: El presente estudio está anidado en el proyecto 1102-04-16433, financiado por COLCIENCIAS y la Universidad Industrial de Santander.

${ }^{1}$ Grupo de Epidemiología Clínica del Centro de Investigaciones Epidemiológicas, Universidad Industrial de Santander (UIS). Bucaramanga, Colombia. ${ }^{2}$ Laboratorio Clínico de la Facultad de Salud UIS. ${ }^{3}$ Departamento de Pediatría, Escuela de Medicina de la UIS.

Correspondencia a: Dr. Fredi Alexander Díaz Quijano. Cra 32 № 29-31, Tercer piso. Centro de Investigaciones Epidemiológicas, Universidad Industrial de Santander. Bucaramanga, Colombia. E mail: fre_diazq@yahoo.com 
$\mathrm{E}^{1}$ dengue es la enfermedad viral transmitida por artrópodos más importante en el mundo ${ }^{1}$ y representa casi la mitad de los casos de síndrome febril agudo (SFA) de origen inaparente que consultan a las instituciones de salud en áreas endémicas ${ }^{2,3}$. En Suramérica se ha registrado el más dramático ascenso en el número de casos en la última década ${ }^{1,4}$, siendo Colombia el país con el mayor número de casos de dengue hemorrágico (DH) en los últimos años ${ }^{5,6}$.

La mayoría de los pacientes que sufren dengue desarrollan una enfermedad febril benigna, conocida como dengue clásico (DC), con alta frecuencia de manifestaciones inespecíficas como mialgias, artralgias, cefalea, dolor retro-ocular, exantema y leucopenia ${ }^{3}$. Una proporción variable de pacientes también desarrolla complicaciones como sangrado espontáneo, trombocitopenia y signos de extravasación plasmática (derrames pleurales, hipoalbuminemia o hemoconcentración). La conjunción de estas alteraciones define el síndrome conocido como DH, que se asocia a una mayor morbilidad y mortalidad $^{1}$.

Aunque no existe una terapia antiviral específica contra el dengue, algunas intervenciones tempranas han mostrado un efecto sobre pronóstico. De esta forma, medidas como la hidratación adecuada, la correcta selección de antipiréticos y la hospitalización temprana de los casos severos, se han asociado a un mejor pronóstico y una menor incidencia de complicaciones potencialmente letales ${ }^{7-9}$.

La alta incidencia del dengue, el riesgo de complicaciones y la utilidad de un manejo inicial adecuado, han justificado la búsqueda de herramientas para el diagnóstico temprano de esta enfermedad y, especialmente, la identificación de indicadores pronósticos que permitan tomar decisiones tras la primera evaluación de los pacientes con SFA por dengue ${ }^{3,10-12}$.

Recientemente, se ha estudiado algunos marcadores de estrés oxidativo en pacientes con dengue y se ha establecido diferencias entre estos pacientes y controles sin procesos infecciosos ${ }^{13,14}$. Sin embargo, estos estudios no han tenido el poder suficiente para identificar variaciones asociadas a la severidad del dengue. Adicionalmente, el análisis se ha limitado a la comparación entre los grupos de DC, DH, pacientes con choque por dengue y DC con manifestaciones hemorrágicas; quedando sin evaluar la asociación entre el estrés oxidativo y cada una de las manifestaciones clínicas y complicaciones específicas de la enfermedad.

La glutatión peroxidasa (GP) es una de las enzimas antioxidantes más importantes y su nivel sérico se ha evaluado ampliamente como un indicador del estrés oxidativo en diferentes procesos infecciosos ${ }^{13-17}$. De esta manera, el objetivo del presente trabajo es describir los niveles de GP en una cohorte de pacientes con dengue y establecer su asociación con los hallazgos clínicos, encontrados en los primeros días de enfermedad, y con las complicaciones detectadas durante el seguimiento.

\section{MÉtodos}

El estudio se desarrolló en el área metropolitana de Bucaramanga, la cual incluye 4 municipios del departamento de Santander, al noreste de Colombia. En este departamento, las tasas de incidencia de dengue han oscilando entre 113,4 y 268,7 casos por 100.000 habitantes, en los últimos años ${ }^{18,19}$.

En el periodo comprendido entre abril de 2003 y diciembre de 2004, se evaluó prospectivamente una cohorte de pacientes mayores de cinco años, con síndrome febril agudo por dengue, de menos de 96 h de evolución, en quienes se logró realizar un seguimiento con un mínimo de: 3 valoraciones médicas, 3 mediciones de hematocrito $y$ un recuento de plaquetas.

Se excluyeron los individuos con enfermedades concomitantes como diabetes, SIDA, desórdenes hematológicos, enfermedad cardiaca 0 tumoral conocida y aquellos que al momento del ingreso ya presentaban complicaciones como DH (ver definición de caso), sangrado mayor, hipoalbuminemia $(<3 \mathrm{~g} / \mathrm{dL})$, derrames serosos o choque.

Todos los pacientes incluidos (o sus padres en los menores de 18 años) firmaron un consentimiento informado. El protocolo de este trabajo fue aprobado por el Comité de Ética de la Facultad de Salud de la Universidad Industrial de Santander.

Captación y seguimiento. La evaluación basal incluyó el registro de signos y síntomas; y la toma de un cuadro hemático (con recuento de plaquetas). Entre las horas 48 y 96 de enfermedad, se 
tomó una muestra de plasma citratado, para la medición de la enzima glutatión peroxidasa, y una de suero para realizar las siguientes pruebas: medición de transferasas hepáticas (AST y ALT), perfil lipídico (colesterol total, HDL, LDL y triglicéridos), un ELISA de captura para IgM específica de dengue y técnicas de aislamiento viral.

El aislamiento se practicó en cultivo de células de mosquito Aedes albopictus (clon C6/36), empleando las muestras de fase aguda, las cuales permanecieron almacenadas a $-70^{\circ} \mathrm{C}$ hasta el momento del cultivo. Los anticuerpos requeridos para la identificación del virus fueron gentilmente donados por el CDC, Dengue Branch Puerto Rico ${ }^{20}$.

Los pacientes se siguieron al menos hasta el séptimo día de enfermedad con medición diaria del hematocrito. El recuento de plaquetas fue repetido diariamente en los casos con recuentos previos inferiores a $120.000 / \mathrm{mm}^{3}$ o al presentarse hemorragias espontáneas, signos de derrames serosos, viraje del hematocrito mayor de $10 \%$ o edemas. A los pacientes que presentaron un recuento de plaquetas inferior a $100.000 / \mu \mathrm{L}$, se les realizó una medición de albúmina en plasma, una ecografía abdominal, bases pulmonares o ambas, en búsqueda de derrames serosos.

Se consideraron casos de DH aquellos pacientes que reunieron los siguientes criterios: un recuento de plaquetas $<100.000 / \mathrm{mm}^{3}$; alguna manifestación hemorrágica espontánea (o al menos una prueba de torniquete positiva) y evidencia de extravasación plasmática, documentada por la presencia de efusión pleural, ascitis, hipoalbuminemia o un viraje del hematocrito mayor de $20 \% 21$. Los pacientes que no reunían los criterios de $\mathrm{DH}$, se clasificaron como DC.

Entre los días 7 y 15 desde el inicio de la fiebre, se realizó una segunda prueba de $\operatorname{IgM}$. La infección por dengue se consideró confirmada cuando hubo un aislamiento viral, un cambio de negativo a positivo en las pruebas pareadas de IgM o un incremento de los títulos de anticuerpos de al menos 4 veces ${ }^{21}$.

Medición de GP. En las muestras de plasma con citrato de sodio $3,2 \%$, tomadas en la primera valoración (entre 48 y $96 \mathrm{~h}$ de enfermedad), se realizó la medición de GP empleando los materiales y reactivos de la casa comercial RANDOX (Kit RS505). Se realizó la lectura a una temperatura de $37^{\circ} \mathrm{C}$ y a una longitud de onda de $340 \mathrm{~nm}$. Se empleó un equipo Microlab 200 de Merck. La concentración de glutation peroxidasa se expresó en U/L

Análisis de datos. Inicialmente, con base en la media de la población estudiada, se dividieron los individuos en dos grupos según los niveles de GP. Posteriormente, mediante análisis bivariado, se evaluaron las asociaciones entre los niveles de GP y las manifestaciones clínicas, reportadas en la evaluación inicial, así como con las complicaciones documentadas durante el seguimiento: sangrado espontáneo, trombocitopenia, extravasación plasmática y DH.

Se empleó la prueba $\mathrm{t}$ de Student para las variables continuas y la prueba de $\mathrm{Chi}^{2}$ para las discretas. Posteriormente, para identificar las hallazgos clínicos asociados de forma independiente con los niveles de GP, se efectuó un análisis de regresión logística que incluyó a todas las variables relacionadas con GP (con $p<0,2$ en el análisis bivariado). Para cada variable se determinó el odds ratio (OR) ajustado y se consideró como asociación estadísticamente significativa aquella con valor $p$ de $<0,05$. En el procesamiento de los datos se empleó el programa estadístico STATA versión 9.2.

\section{RESULTADOS}

Durante el periodo del estudio, se captaron 161 pacientes con dengue, cuya edad osciló entre los 6 y los 85 años (media: 29,2; desviación estándar: 16,2 años). Se observó un leve predominio del género femenino, representando a $53,4 \%$ del total (86/161). Al ingreso, el tiempo de evolución del síndrome febril agudo fue, en promedio, de 73,52 h (IC 95\%: 70,6-76,44) y estuvo acompañado principalmente por cefalea, mialgias, artralgias, escalofrío e hiporexia, síntomas presentes en más de $80 \%$ de los casos (Tabla 1 ).

Del total, $75(46,6 \%)$ pacientes presentaron hemorragia espontánea, $40(24,8 \%)$ desarrollaron trombocitopenia $(<100.000$ plaquetas $/ \mu \mathrm{L})$ y 28 $(17,4 \%)$ mostraron algún signo de extravasación plasmática, siendo el viraje del hematocrito el más frecuente. Durante seguimiento, 12 pacientes reunieron estos criterios de severidad, configurando 
Tabla 1. Características clínicas de los pacientes con dengue incluidos y según el nivel de G P.

\begin{tabular}{|c|c|c|c|c|}
\hline Variable & $\begin{array}{c}\text { Total } \\
(\mathrm{n}=161)\end{array}$ & $\begin{array}{c}\mathrm{GP}>1200 \\
\mathrm{U} / \mathrm{L}(\mathrm{n}=82)\end{array}$ & $\begin{array}{c}\mathrm{GP} \leq 1200 \\
\mathrm{U} / \mathrm{L}(\mathrm{n}=79)\end{array}$ & $\begin{array}{c}\text { Valor } \\
\mathrm{p}\end{array}$ \\
\hline Sexo masculino - No (\%) & $75(46,6)$ & $33(40,2)$ & $42(53,2)$ & 0,10 \\
\hline Edad media $\pm \mathrm{DE}$ & $29,23 \pm 16,23$ & $27,2 \pm 14,32$ & $31,34+17,85$ & 0,11 \\
\hline \multicolumn{5}{|l|}{ Manifestaciones - No (\%) } \\
\hline Cefalea & $151(93,8)$ & $80(97,6)$ & $71(89,9)$ & 0,04 \\
\hline Dolor retroocular & $111(68,9)$ & $59(72)$ & $52(65,8)$ & 0,40 \\
\hline Mialgias & $149(92,6)$ & $78(95,1)$ & $71(89,9)$ & 0,20 \\
\hline Artralgias & $133(82,6)$ & $73(89,0)$ & $60(76)$ & 0,03 \\
\hline Escalofrío & $154(95,7)$ & $78(95,1)$ & $76(96,2)$ & 0,74 \\
\hline Hiporexia & $145(90,0)$ & $73(89,0)$ & $72(91,1)$ & 0,65 \\
\hline Odinofagia & $69(42,9)$ & $34(41,5)$ & $35(44,3)$ & 0,72 \\
\hline Tos & $62(47)$ & $30(39,5)$ & $32(57,1)$ & 0,04 \\
\hline Rinorrea & $50(38,2)$ & $29(38,7)$ & $21(37,5)$ & 0,89 \\
\hline Exantema & $61(45,5)$ & $38(50)$ & $23(39,7)$ & 0,23 \\
\hline Vómito & $44(27,3)$ & $24(29,3)$ & $20(25,3)$ & 0,57 \\
\hline Diarrea & $48(29,8)$ & $22(26,8)$ & $26(32,9)$ & 0,40 \\
\hline Dolor abdominal & $84(52,2)$ & $45(54,9)$ & $39(49,4)$ & 0,48 \\
\hline Fiebre $\left(\mathrm{T}^{\mathrm{o}} \geq 38^{\circ} \mathrm{C}\right)$ & $20(12,4)$ & $6(7,3)$ & $14(17,7)$ & 0,045 \\
\hline Deshidratación & $68(56,4)$ & $37(48,7)$ & $31(56,4)$ & 0,39 \\
\hline Eritema facial & $100(62,1)$ & $53(64,6)$ & $47(59,5)$ & 0,51 \\
\hline Prueba torniquete & $76(47,5)$ & $45(54,9)$ & $31(39,7)$ & 0,06 \\
\hline Sangrado espontáneo & $24(14,9)$ & $14(17,1)$ & $10(12,7)$ & 0,43 \\
\hline \multicolumn{5}{|l|}{ Complicaciones*: } \\
\hline Sangrado espontáneo & $75(46,6)$ & $45(54,9)$ & $30(38)$ & 0,03 \\
\hline Fuga plasmática $^{\dagger}$ & $28(17,4)$ & $14(17,1)$ & $14(17,7)$ & 0,91 \\
\hline Viraje de Hto $\geq 20 \%$ & $25(15,5)$ & $14(17,1)$ & $11(13,9)$ & 0,58 \\
\hline Trombocitopenia & $40(24,8)$ & $24(29,3)$ & $16(20,3)$ & 0,19 \\
\hline $\mathrm{DH}$ & $12(7,45)$ & $4(4,88)$ & $8(10,13)$ & 0,20 \\
\hline
\end{tabular}

*Documentadas durante el seguimiento.

${ }^{\dagger}$ Definida como la presencia de cualquiera de los siguientes signos: viraje del hematocrito (Hto) $\geq 20 \%$, derrames pleurales, ascitis o hipoalbuminemia.

el síndrome de dengue hemorrágico (incidencia $=7,32 \%$ ) (Tabla 1).

En la población evaluada, la enzima glutatión peroxidasa (GP) mostró un valor promedio de 1.197,6 U/L (IC 95\%: 1.089,5-1.305,7). Los niveles de este marcador de estrés oxidativo fueron significativamente más altos en los pacientes que presentaron algún sangrado espontáneo $(p=0,03)$. Sin embargo, la GP no se asoció con los otros signos de severidad de la enfermedad ( $p>0,15$, para todas las variables) (Tabla 1).
Con base en el promedio de la GP, se seleccionó el valor de $1.200 \mathrm{U} / \mathrm{L}$ como punto de corte para dividir la población en dos grupos: 82 con niveles de GP superiores y 79 con valores iguales o menores. Cuando se compararon las manifestaciones clínicas de los pacientes con dengue de acuerdo a los niveles de GP, se observó que aquellos con más de 1.200 U/L de GP presentaban mayor frecuencia de cefalea $(p$ $=0,04)$ y artralgias $(p=0,03)$; menor frecuencia de tos $(p=0,04)$ y fiebre al examen físico $(p<0,05)$; 
frecuencia cardíaca superior ( $p=0,0006$ ) y presión arterial sistólica (PAS) significativamente inferior ( $p=0,009$ ) (Tablas 1 y 2).

Por otra parte, no se encontró asociación entre la GP y la mayoría de los marcadores bioquímicos evaluados, entre ellos, las transaminasas hepáticas, el colesterol y las lipoproteínas de alta y baja densidad. Sin embargo, se observó una asociación negativa entre los niveles de triglicéridos y los valores de GP (Tabla 3).

Estos hallazgos fueron evaluados en un análisis de regresión logística que incluyó las variables asociadas con los niveles de GP (con p <0,20), entre las que se cuentan la edad y el género. En este modelo, se observó que el síntoma de artralgias, la fiebre y la frecuencia cardíaca en decúbito, estuvieron asociadas de forma independiente al resultado de esta prueba (Tabla 4).

\section{Discusión}

En otros estudios se evidencia que las complicaciones de esta arbovirosis y la necesidad de hospitalizar, suelen aparecer hacia el quinto día desde el inicio de los síntomas ${ }^{22}$. En este trabajo se captaron pacientes con dengue en un momento relativamente temprano de la enfermedad. De esta manera, los cambios de la GP detectados en los primeros días y las asociaciones evidenciadas ubican al estrés oxidativo en la patogénesis temprana del dengue.

Tabla 2. Análisis de variables continuas según el nivel de G P en pacientes con dengue

\begin{tabular}{|lccccc|}
\hline Variable* & $\begin{array}{c}\text { GP }>1200 \\
(\mathrm{n}=82)\end{array}$ & IC $95 \%$ & $\begin{array}{c}\text { GP } \leq 1200 \\
(\mathrm{n}=79)\end{array}$ & IC 95\% & $\begin{array}{c}\text { Valor } \\
\mathrm{p}\end{array}$ \\
\hline Peso & 59,80 & $56,81-62,8$ & 61,58 & $57,67-65,49$ & 0,47 \\
FR & 19,06 & $18,42-19,7$ & 18,9 & $18,01-19,79$ & 0,77 \\
Temperatura & 36,87 & $36,66-37,08$ & 36,63 & $36,40-36,87$ & 0,14 \\
FC & 84,95 & $81,8-88,11$ & 77,53 & $74,75-80,31$ & 0,0006 \\
PAS & 113,61 & $110,82-116,4$ & 119,87 & $116,09-123,66$ & 0,009 \\
PAD & 71,5 & $69,34-73,66$ & 73,05 & $70,78-75,32$ & 0,33 \\
Plaquetas $\times 10^{3} / \mu \mathrm{L}$ & 143,6 & $129,72-157,5$ & 148,8 & $132,02-165,57$ & 0,64 \\
\hline
\end{tabular}

*FR: frecuencia respiratoria; FC: frecuencia cardíaca; PAS: presión arterial sistólica; PAD: presión arterial diastólica.

Tabla 3. Variables bioquímicas según el nivel de $\mathrm{G}$ P en pacientes con dengue

\begin{tabular}{|c|c|c|c|c|c|}
\hline Variable* & $\begin{array}{c}\mathrm{GP}>1200 \\
\mathrm{U} / \mathrm{L} \\
(\mathrm{n}=82)\end{array}$ & IC $95 \%$ & $\begin{array}{c}\mathrm{GP} \leq 1200 \\
\mathrm{U} / \mathrm{L} \\
(\mathrm{n}=78)\end{array}$ & IC $95 \%$ & $\begin{array}{c}\text { Valor } \\
\mathrm{p}\end{array}$ \\
\hline AST (U/L) & 99,71 & $79,63-119,78$ & 107,6 & $87,36-127,83$ & 0,58 \\
\hline ALT (U/L) & 77,81 & $60,56-95,06$ & 85 & 63,87-106,13 & 0,60 \\
\hline Colesterol (mg/dL) & 142,7 & $133,95-151,45$ & 149,10 & $140,42-157,78$ & 0,30 \\
\hline HDL (U/L) & 36,79 & $34,36-39,21$ & 35,73 & $33,65-37,80$ & 0,51 \\
\hline LDL (U/L) & 83,32 & $75,9-90,74$ & 85,48 & $78,02-92,95$ & 0,68 \\
\hline Triglicéridos (mg/dL) & 114,57 & $101,87-127,28$ & 140,43 & $119,18-161,67$ & 0,04 \\
\hline
\end{tabular}

*AST: aspartato amino transferasa; ALT: alanito amino transferasa, HDL: lipoproteína de alta densidad; LDL: lipoproteína de baja densidad. 
Tabla 4. Variables asociadas de forma independiente a un nivel de GP >1200 U/L. Análisis de regresión logística*

\begin{tabular}{|llrc|}
\hline Variable $^{\dagger}$ & OR & $($ IC 95\%) & Valor $\mathrm{p}$ \\
\hline Edad (cada 10 años) & 0,92 & $(0,69-1,22)$ & 0,55 \\
Género masculino & 1,11 & $(0,5-2,49)$ & 0,79 \\
Cefalea & 2,98 & $(0,44-20,38)$ & 0,27 \\
Artralgias & 3,1 & $(1,05-9,14)$ & 0,04 \\
Tos & 0,6 & $(0,27-1,31)$ & 0,20 \\
Fiebre (To $\left.\geq 38^{\circ} \mathrm{C}\right)$ & 0,14 & $(0,04-0,5)$ & 0,003 \\
Prueba de torniquete & 2,13 & $(0,95-4,81)$ & 0,07 \\
Sangrado espontáneo & 1,34 & $(0,41-4,32)$ & 0,63 \\
Trombocitopenia & 1,25 & $(0,51-3,1)$ & 0,63 \\
FC (cada 10 latidos/min) & 1,59 & $(1,18-2,14)$ & 0,002 \\
PAS (cada 10 mmHg) & 0,75 & $(0,56-1,01)$ & 0,06 \\
Triglicéridos (cada 10 mg/dL) & 0,95 & $(0,89-1,01)$ & 0,09 \\
\hline
\end{tabular}

*Se incluyeron todas las variables con $\mathrm{p}<0,20$ en el análisis bivariado.

${ }^{\dagger}$ Para las variables continuas, se muestra la asociación con cada aumento de 10 en las unidades respectivas.

${ }^{\ddagger}$ Recuento de plaquetas $\leq 100.000 / \mu \mathrm{L}$.

La actividad antioxidante de la GP evita una cadena de reacciones de peroxidación lipídica y con ello, los efectos tóxicos de los radicales libres ${ }^{13,15}$. Varios estudios han evidenciado una disminución estadísticamente significativa de esta enzima asociada a la infección de diferentes patógenos $^{16,17}$. Este cambio no ha sido claramente comprendido, sin embargo, experimentos en modelos animales, donde los niveles de GP decrecen tras la inoculación de patógenos ${ }^{23,24}$, sugieren que esta disminución podría corresponder a un consumo de la enzima como parte integral de la respuesta natural a la infección. De forma análoga, cuando se han evaluado pacientes con dengue, éstos presentan niveles de GP significativamente inferiores a los encontrados en controles sanos ${ }^{13,14}$.

Sin embargo, cuando se han comparado los casos de dengue entre sí, de acuerdo a la presencia de sus complicaciones, se ha observado que los niveles de GP son mayores cuanto más severa es la enfermedad ${ }^{13,14}$. Aunque los anteriores estudios no demostraron que esta relación fuera estadísticamente significativa, ello podría explicarse por un insuficiente tamaño de la muestra 0 por el hecho de que la enzima fue medida durante fases tardías de la enfermedad, cuando la fuga plasmática es más pronunciada y la medición de las proteínas séricas es menos confiable.
En el presente trabajo, en un grupo de pacientes con dengue, los niveles más altos de GP medidos tempranamente estuvieron asociados a algunas complicaciones de la enfermedad. A manera de hipótesis, planteamos que en los casos severos no ocurre el consumo esperado de GP y esto podría representar una inadecuada actividad antioxidante. Esta condición podría permitir que el estrés oxidativo ocurra con mayor intensidad y aumenten sus efectos nocivos.

La fisiopatología del sangrado espontáneo no ha sido esclarecida en su totalidad. Hasta el momento, la trombocitopenia y las alteraciones de la coagulación han sido los principales factores relacionados con las complicaciones hemorrágicas $^{19,25,26}$. En este estudio, la GP se encontró elevada tempranamente en los pacientes que desarrollaron hemorragias espontáneas, hallazgo que representa un nuevo aspecto no explorado previamente en la patogénesis del sangrado en dengue y que podría estar relacionado con el efecto deletéreo que ocasionan los radicales libres de oxígeno sobre los tejidos ${ }^{27}$.

Los niveles mayores de GP también se vieron relacionados con una mayor frecuencia de síntomas característicos del dengue, como son la cefalea y las artralgias. Este hallazgo sugiere que el estrés oxidativo tiene un papel en la expresión 
de la enfermedad en su fase febril aguda, aun antes de que se desarrollen las complicaciones más temidas del dengue.

Por otra parte, aunque la GP no se asoció estadísticamente con los signos de extravasación plasmática, este marcador de estrés oxidativo mostró una fuerte asociación con los cambios hemodinámicos relacionados con este fenómeno vascular. De esta forma, niveles altos de GP se asociaron a mayor frecuencia cardiaca y menor presión arterial sistólica, cambios que podrían estar relacionados con disminución del volumen intravascular. Este supuesto también podría explicar la consistente asociación negativa entre los niveles de GP y la temperatura, dado que una inadecuada perfusión, en lugar de una ausencia de fiebre, podría ser la base de este hallazgo.

Finalmente, otro resultado interesante fue la asociación negativa entre la GP y los triglicéridos. Aunque este hallazgo no se ha descrito anterior-

\section{REFERENCIAS}

1. Stephenson JR. The problem with dengue. Trans R Soc Trop Med Hyg 2005; 99: 643-6.

2. Silarug N, Foy HM, Kupradinon S, Rojanasuphot S, Nisalak A, Pongsuwant Y. Epidemic of fever of unknown origin in rural Thailand, caused by influenza A (H1N1) and dengue fever. Southeast Asian J Trop Med Public Health 1990; 21: 61-7.

3. Díaz FA, Martínez RA, ViLar LA. Criterios clínicos para diagnosticar el dengue en los primeros días de enfermedad. Biomédica 2006; 26: 22-30.

4. Siqueira JB Jr, Marteli CM, Coelho GE, Simplicio AC, HATCH DL. Dengue and dengue hemorrhagic fever, Brazil, 1981-2002. Emerg Infect Dis 2005; 11: 48-53.

5. Pan American Health Organization. 2003: Number of Reported Cases of Dengue \& Dengue Hemorrhagic Fever (DHF), Region of the Americas (by country and subregion). Disponible en: http:// www.paho.org/English/AD/DPC/CD/dengue-cases-2003.htm

6. Pan American Health Organization. 2004: Number of Reported Cases of Dengue \& Dengue Hemorrhagic Fever (DHF), Region of the Americas (by country and subregion). Disponible en: http:// mente en dengue, es concordante con las observaciones realizadas por otros autores, quienes sugieren que el estrés oxidativo generaría una disminución de los lípidos séricos, pues éstos son altamente susceptibles a los efectos tóxicos de los radicales libres ${ }^{13}$.

En conclusión, los resultados del presente estudio sugieren que los niveles de GP están relacionados con la expresión de algunas manifestaciones tempranas del dengue y con la posterior aparición de sangrado espontáneo. Lo anterior plantea que el estrés oxidativo podría hacer parte de los mecanismos iniciales asociados a la presentación de la enfermedad y, adicionalmente, sus marcadores podrían ser predictores de complicaciones como el sangrado espontáneo. De otro lado, estos hallazgos soportan la búsqueda de agentes terapéuticos basados en la regulación del estrés oxidativo para prevenir las complicaciones del dengue.

www.paho.org/English/AD/DPC/CD/dengue-cases-2004.htm

7. Díaz-Quijano FA, Viliar-Centeno LA, Martínez-Vega RA. Efecto de la administración de dipirona sobre la gravedad del dengue en una cohorte prospectiva. Enferm Infecc Microbiol Clin 2005; 23: 593-7.

8. Harris E, Pérez L, Phares C, Pérez M, Idiaquez W, RochA J ET AL. Fluid Intake and decreased risk for hospitalization Dengue Fever, Nicaragua. Emerging Infectious Diseases 2003; 9: 1003-6.

9. Chairulfatah A, Setiabudi D, Ridad A, Colebunders R. Clinical manifestations of dengue haemorrhagic fever in children in Bandung, Indonesia. Ann Soc Belg Med Trop 1995; 75: 291-5.

10. Kalayanarooj S, Vaughn DW, Nimmannitya S, Green S, SuntaYAKorn S, KunENTRASAi N et al. Early Clinical and Laboratory Indicators of Acute Dengue Illness. J Infect Dis 1997; 176: 313-21.

11. Díaz-Quijano FA, Viliar-Centeno LA, Martínez-Vega RA. Indicadores tempranos de infección por dengue en niños. An Pediatr (Barc) 2006; 64: 523-9.

12. Díaz-Quijano FA, Martínez-Vega RA, Viliar-Centeno LA. Indicadores de severidad en dengue. Enferm Infecc Microbiol Clin 2005; 23: 529-32.

13. Ray G, Kumar V, Kappor AK Dutta AK, Batra S. Status of antioxidants and other biochemical 
abnormalities in children with dengue fever. J Trop Pediatr 1999; 45: 4-7.

14. Gil L, Martínez G, Tapanes R, Castro O, González D, BernaRDo L ET al. Oxidative stress in adult dengue patients. Am J Trop Med Hyg 2004; 71: 652-7.

15. Pabón A, Carmona J, Burgos LC, Blair S. Oxidative Stress in patients with non-complicated malaria. Clin Biochem 2003; 36: 71-8.

16. Wen J, Yachelini PC, Sembaj A, Manssur RE, Garg NJ. Increased oxidative stress is correlated with mitochondrial dysfunction in chagasic patients. Free Radical Biol Med 2006; 41: 270-6.

17. Levent G, Al A, Ahmet A, Polat EC, Aytaç Ç, Ayse E ET AL. Oxidative stress and antioxidant defense in patients with chronic hepatitis $\mathrm{C}$ patients before and after pegylated interferon alfa-2b plus ribavirin therapy. Journal of Translational Medicine 2006; 4: 25.

18. SIVIGILA. Comportamiento por regiones del dengue en el 2001. Boletín Epidemiológico Semanal. Semana epidemiológica № 02. Enero 06 a 12 de 2002. www.col.ops-oms.org/sivigila/2002/BOLE02_02.htm

19. SIVIGILA. Enfermedades de notificación obligatoria. Informe primer semestre de 2003. Boletín Epidemiológico Semanal. Semana epidemiológica No. 26. Junio 22 - 28 de 2003. www.col.opsoms.org/sivigila/2003/BOLE26 03.pdf

20. Martínez-Vega RA, Díaz-Quijano FA, Viliar-Centeno LA. Dificultad para el diagnóstico clínico temprano del dengue en un área endémica y su impacto sobre el manejo inicial. Rev Méd Chile 2006; 134: 1153-60.
21. Organización Panamericana de la Salud. Definiciones de casos. Dengue. Boletín Epidemiológico 2000; 21: 14-5.

22. Díaz-Quijano FA, Vimar-Centeno LA, Martínez-Vega RA. Complicaciones asociadas a la trombocitopenia profunda en pacientes con Dengue. Rev Méd Chile 2006; 134: 167-73.

23. MohankUmar K, RAmasamy P. White spot syndrome virus infection decreases the activity of antioxidant enzymes in Fenneropenaeus indicus. Virus Res 2006; 115: 69-75.

24. Kolodziejczyk L, Siemieniuk E, Skrzydlewska E. Antioxidant potential of rat liver in experimental infection with Fasciola hepatica. Parasitol Res 2005; 96: 367-72.

25. Chua MN, Molanida R, de Guzmán M, Laberiza F. Prothrombin time and partial thromboplastin time as a predictor of bleeding in patients with dengue hemorrhagic fever. Southeast Asian J Trop Med Public Health 1993; 24 Suppl 1: 141-3.

26. Wils BA, Oragui EE, Stephens AC, Daramola OA, Dung NM, LOAN HT ET AL. Coagulation abnormalities in dengue hemorrhagic Fever: serial investigations in 167 Vietnamese children with Dengue shock syndrome. Clin Infect Dis 2002; 35: 277-85.

27. CÉspedes T, Sánchez SerRano DI. Algunos aspectos sobre el estrés oxidativo, el estado antioxidante y la terapia de suplementación. Instituto de Cardiología y Cirugía Cardiovascular. Rev Cubana Cardiol 2000; 14: 55-60.

Agradecimientos

Los autores agradecemos al Dr. Alejandro Ceballos, del Departamento de Salud Animal de la Universidad de Caldas, por su asesoría en la medición de la enzima glutatión peroxidasa. También agradecemos a la Dra. Raquel Ocazionez y a los bacteriólogos Fabián Cortés y Sergio Gómez, del Centro de Investigaciones en Enfermedades Tropicales (CINTROP), por su colaboración en la realización de las pruebas para la confirmación de la infección por dengue. 